\title{
Presence of Extra $P_{11}$ Resonances in Zagreb Analysis Since 1995.
}

\author{
S. CECI, A. ŠVARC and B. ZAUNER \\ Rudjer Bošković Institute, \\ Bijenička c. 54, \\ 10000 Zagreb, Croatia \\ E-mail: Alfred.Svarc@irb.hr
}

December 6, 2018

\begin{abstract}
The partial wave T-matrices for the $\pi \mathrm{N}, \eta \mathrm{N}$ and $\pi^{2} \mathrm{~N}$ channels have been obtained within the framework of the coupled channel model using the $\pi \mathrm{N}$ elastic and $\pi \mathrm{N} \rightarrow \eta \mathrm{N}$ data base as input. It has been shown that for the $P_{11}$ partial wave an equally good representation of the experimental data (namely the $T_{\pi N, \pi N}$ and $T_{\pi N, \eta N}$ T-matrices) can be obtained using either three, or four poles for the Green function propagator. However, the three Green function pole solution is not acceptable due to the structure of the extracted resonances. The two out of four $P_{11}$ resonances, those lying in the energy range $1700 \mathrm{MeV}$ $<M_{R}<1800 \mathrm{MeV}$, are poorly determined, but they seem to be strongly inelastic. The inclusion of other inelastic channels is needed to determine masses and widths of missing resonances with greater precision.
\end{abstract}

The partial wave T-matrices for the $\pi \mathrm{N}, \eta \mathrm{N}$ and $\pi^{2} \mathrm{~N}$ channels have been obtained within the framework of the three body coupled channel model (CMU-LBL) using the $\pi \mathrm{N}$ elastic and $\pi \mathrm{N} \rightarrow \eta \mathrm{N}$ data base as input [1, 2]. As it has been shown in Fig.1 the number of Green function poles (N), the respective pole positions $\left(s_{i}\right)$ and the channel-resonance mixing parameters $\left(\gamma_{a i}\right)$ are the input parameters of the fitting procedure which are adjusted in such a way that the experimental input ( $\pi \mathrm{N}$ elastic T-matrices and $\pi \mathrm{N} \rightarrow \eta \mathrm{N}$ data base) is well reproduced. In reality, the number of T-matrix poles (the Green function poles $s_{i}$ ), which are to be interpreted as resonant states, is 
chosen in advance, and the only criterion is the quality of data reproduction. The schematic representation of the fitting procedure is shown with full lines in Fig.1. Only upon obtaining the full coupled channel T-matrices set which satisfactory reproduces the experimental data, the resonance parameters are extracted from the final set of obtained T-matrices. Let us emphasize that the resonance parameters are extracted a posteriori. We have no influence upon the type of resonance which we are going to obtain during the fitting procedure, we just know their number, namely, the number of Green function poles.

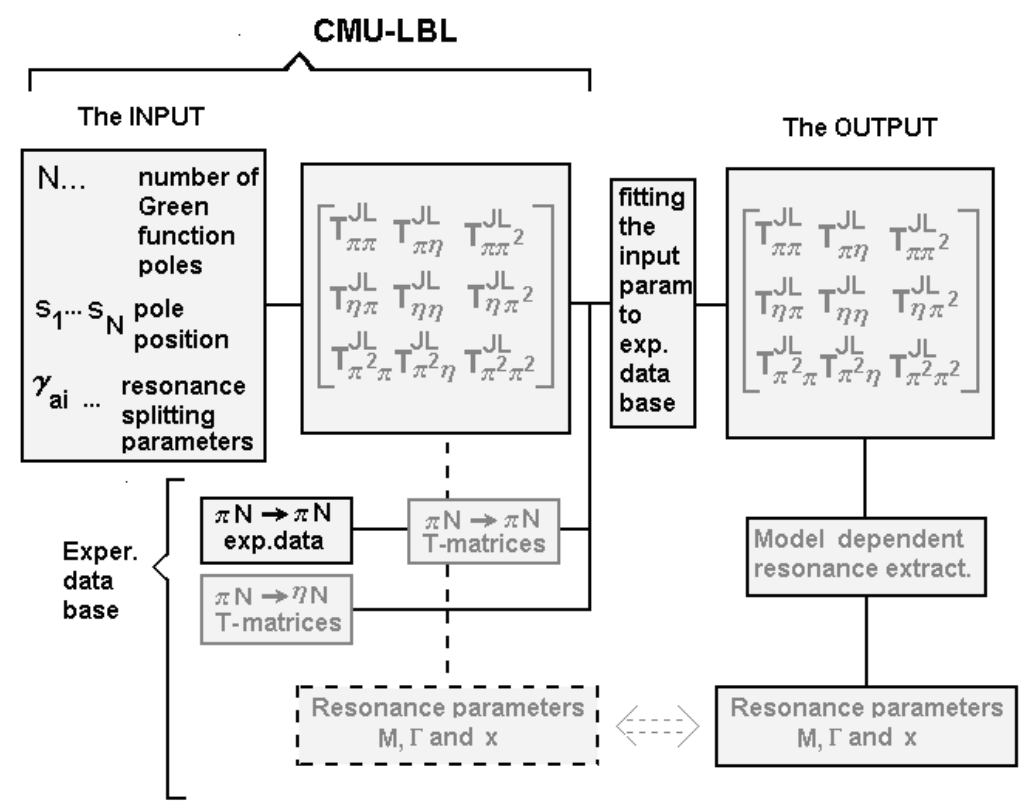

Figure 1: The schematic representation of resonance extraction in the coupled channel formalism (CMU-LBL). The full lines represent the present situation, the dashed lines are the suggested and needed modifications.

As it is shown in Fig.2. the same quality of the fit to the input experimental data base, which is reflected through almost identical form of the $T_{\pi N, \pi N}$ and $T_{\pi N, \eta N}$, is obtained with three $(\mathrm{N}=3$, thin solid line) and four $(\mathrm{N}=4$, thick solid line) poles in the Green function, respectively. However, as it is shown in Fig. 3 the prediction for the remaining coupled channel T-matrices $T_{\eta N, \eta N}$ and $T_{\pi^{2} N, \pi^{2} N}$ is dramatically different for the three and four pole solution (thick and thin line). The resonance parameters, extracted from both solutions are given in Table 1.

We claim that the obtained three body solution is not acceptable because it shows strong coupling of second and third resonance to the $\eta \mathrm{N}$ channel 

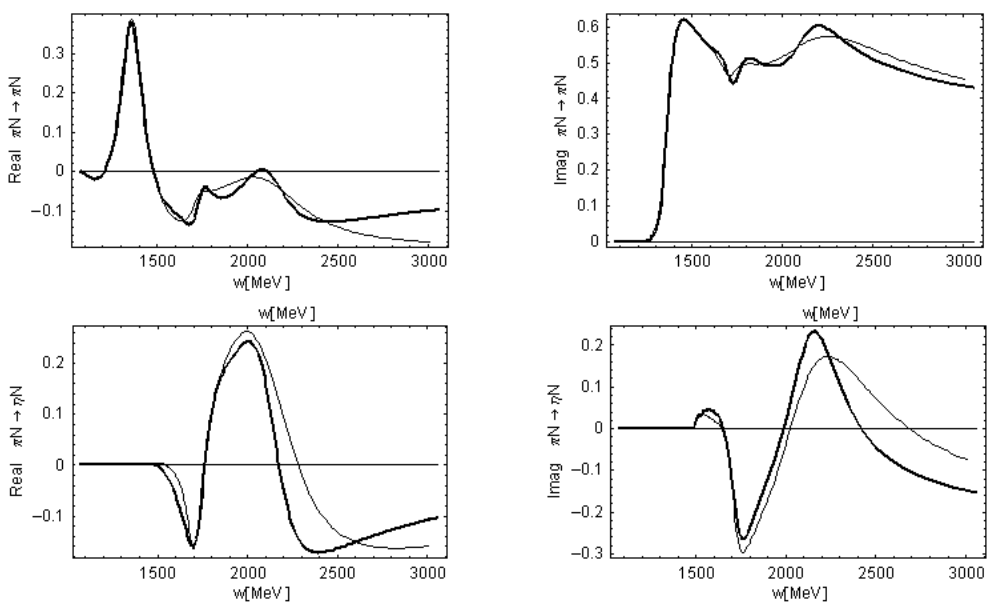

Figure 2: The $P_{11}$ partial wave T-matrices for three (thin lines) and four (thick lines) T-matrix poles for $\pi \mathrm{N} \rightarrow \pi \mathrm{N}$ and $\pi \mathrm{N} \rightarrow \eta \mathrm{N}$ processes.
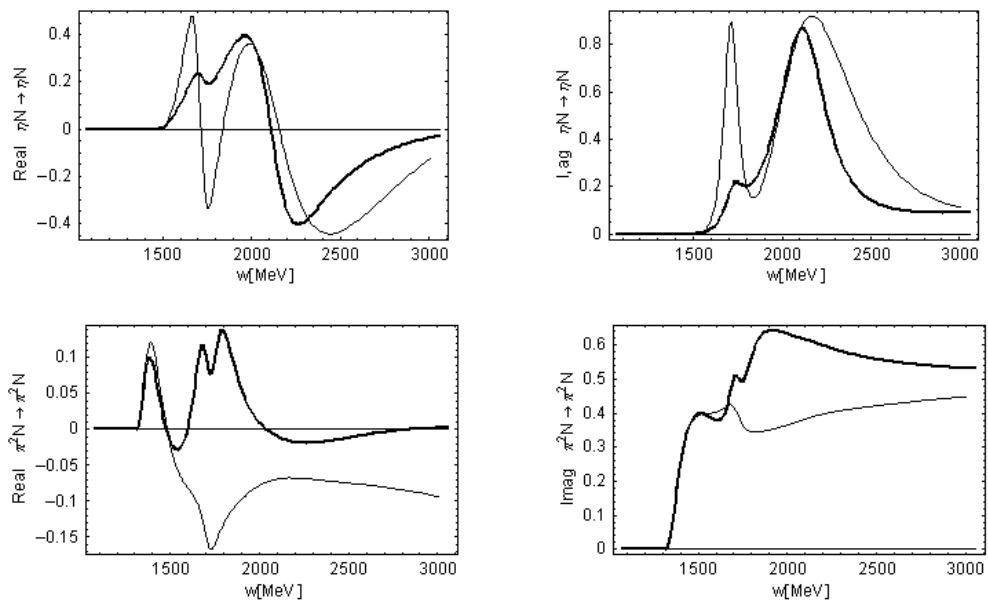

Figure 3: The $P_{11}$ partial wave T-matrices for three (thin lines) and four (thick lines) T-matrix poles for $\eta \mathrm{N} \rightarrow \eta \mathrm{N}$ and $\pi^{2} \mathrm{~N} \rightarrow \pi^{2} \mathrm{~N}$ processes.

only, and shows no branching ration to the third, effective channel which includes processes like $\pi \mathrm{N} \rightarrow \pi \pi \mathrm{N}$ and $\pi \mathrm{N} \rightarrow \mathrm{K} \Lambda$ which are experimentally firmly established.

We offer two alternative explanations: either our fitting procedure is technically inadequate to find a better three pole solution, or we indeed need four resonances in the $P_{11}$ partial wave, second and third strongly inelastic, exactly as indicated in Table 1. 
Resonance parameters for the three and four pole solution.

\begin{tabular}{|c|c|c|c|c|c|}
\hline \multirow{2}{*}{$\begin{array}{c}\text { States } \\
\mathrm{L}_{2 I, 2 J} \\
\left(\begin{array}{l}\mathrm{x}_{\mathrm{el}} \\
\text { Mass/Width }\end{array}\right)\end{array}$} & \multicolumn{5}{|c|}{ Three poles in the Green function } \\
\hline & $\begin{array}{c}\text { Mass } \\
(\mathrm{MeV})\end{array}$ & $\begin{array}{l}\text { Width } \\
(\mathrm{MeV})\end{array}$ & $\begin{array}{c}x_{\pi} \\
(\%) \\
\end{array}$ & $\begin{array}{c}x_{\eta} \\
(\%)\end{array}$ & $\begin{array}{l}x_{\pi^{2}} \\
(\%)\end{array}$ \\
\hline$\overline{\mathrm{P}_{11}\left(\begin{array}{l}51 \\
144 / 135\end{array}\right)}$ & $1426(25)$ & $287(53)$ & 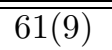 & $0(0)$ & $39(9)$ \\
\hline $\mathrm{P}_{11}\left(\begin{array}{l}12 \\
1710 / 120\end{array}\right)$ & 1724(35) & $116(47)$ & $5(5)$ & $89(7)$ & $6(5)$ \\
\hline $\mathrm{P}_{11}$ & - & - & - & - & - \\
\hline $\mathrm{P}_{11}\left(\begin{array}{c}9 \\
2100 / 200\end{array}\right)$ & $2175(89)$ & $659(207)$ & $9(4)$ & $89(3)$ & $2(2)$ \\
\hline States & \multicolumn{5}{|c|}{ Four poles in the Green function } \\
\hline $\begin{array}{c}\mathrm{L}_{2 I, 2 J} \\
\left(\begin{array}{c}\mathrm{x}_{\mathrm{el}} \\
\text { Mass/Width }\end{array}\right)\end{array}$ & $\begin{array}{l}\text { Mass } \\
(\mathrm{MeV})\end{array}$ & $\begin{array}{l}\text { Width } \\
(\mathrm{MeV})\end{array}$ & $\begin{array}{c}x_{\pi} \\
(\%)\end{array}$ & $\begin{array}{c}x_{\eta} \\
(\%)\end{array}$ & $\begin{array}{l}x_{\pi^{2}} \\
(\%)\end{array}$ \\
\hline$\overline{\mathrm{P}_{11}\left(\begin{array}{l}51 \\
1440 / 135\end{array}\right)}$ & $\overline{1439(19)}$ & $\overline{437(141)}$ & $62(4)$ & $0(0)$ & $38(4)$ \\
\hline $\mathrm{P}_{11}\left(\begin{array}{l}12 \\
1710 / 120\end{array}\right)$ & $1729(16)$ & $180(17)$ & $22(24)$ & $6(8)$ & $72(23)$ \\
\hline $\mathrm{P}_{11}$ & $1740(11)$ & $140(25)$ & $28(34)$ & $12(9)$ & $60(35)$ \\
\hline $\mathrm{P}_{11}\left(\begin{array}{c}9 \\
2100 / 200\end{array}\right)$ & $2157(42)$ & $355(88)$ & $16(5)$ & $83(5)$ & $1(1)$ \\
\hline
\end{tabular}

We conclude that using $\pi \mathrm{N}$ elastic and $\pi \mathrm{N} \rightarrow \eta \mathrm{N}$ data base is, at the present moment, insufficient even to determine the $\eta \mathrm{N}$ elastic channel.

In order to improve the fitting technique, we propose to include the resonance parameters as the "quasi input" into the fitting procedure (dashed lines in Fig.1). That would enable us to search for a particular type of a three body solution, namely solution which reproduces the experimental data set simultaneously with imposing that one of the resonances is inelastic in other then $\eta \mathrm{N}$ channel. If such a procedure fails in the end, the statement that we need more then three resonances in a $P_{11}$ partial wave is fully justified.

\section{References}

[1] R.E. Cutkosky et. al., Phys. Rev. D20, 2804 (1979); R.E. Cutkosky, C.P. Forsyth, R.E. Henrick and R.L. Kelly, Phys. Rev. D20, 2839 (1979); R.L. Kelly and R.E. Cutkosky, Phys. Rev. D20, 2872 (1979).

[2] M. Batinić, I. Dadić, I. Šlaus, A. Švarc, B.M.K. Nefkens and T.S.-H. Lee, Physica Scripta 58, 15 (1998) 